\title{
23 Linking Ecology and Epidemiology: The Case of Infected Resource
}

Sanja Selaković, Peter C. de Ruiter, and Hans Heesterbeek

\subsection{Introduction}

Interspecific interactions in ecological communities are the main mechanisms that determine structure, functioning, and stability of ecosystems (May, 1972, 1973; Neutel et al., 2002; Alessina and Tang, 2012; Mougi and Kondoh, 2012, 2014). These interactions can be qualitatively positive, negative, or neutral, and pairs of these interactions between two species may be of opposite sign (e.g., trophic, parasitic) or of equivalent sign (e.g., mutualistic, competitive). Most of the research on ecological interactions has focused on feeding relations (Odum, 1971; Pimm, 1982; Levin et al., 2009; McCann, 2011; Moore and de Ruiter, 2012), but in recent studies of ecological communities this was extended to parasitic (Huxham et al., 1995; Thompson et al., 2004; Lafferty et al., 2006; Kuris et al., 2008) and non-parasitic non-trophic relations (Thebault and Fountaine, 2010; Fontaine et al., 2011; Kéfi et al., 2012; Mougi and Kondoh, 2012; Sauve et al., 2014).

In this chapter, we focus on parasitic relations and notably on the question of how trophic interactions and infectious agents mutually influence each other. Here we will refer to the combined classes of infectious species as parasites (see next section for details). The impact of parasites in an ecological community can be quantified through their direct influence on the food-web structure, as well as more indirectly through the way they influence physiological traits of host species and trophic relations of the host and non-host species (Kéfi et al., 2012; Selakovic et al., 2014). In this chapter we first briefly discuss the diversity of parasitic interactions, their relationships with host and non-host species, as well as their effects on a simple consumer-resource relationship consisting of one host and one non-host species. The largest part of the chapter is devoted to exploring a basic model, to show how intricately ecological and epidemiological effects are interwoven, even in the simplest possible ecosystem consisting of two species. Even though this model is basic in the sense that it is low dimensional and not meant to realistically represent any particular system, the analysis does hint at broader ecological insight, for example into possible differences between terrestrial and aquatic ecosystems based on parasitic interaction. The simple analysis highlights the need to study the link between ecology and infectious disease epidemiology in more realistic models. 


\subsection{Parasitic Interactions, Diversity, Types, Functional Roles, and Modeling}

The relationship between parasites and their hosts can have aspects of both trophic and non-trophic interactions. Parasites are in essence consumers of resources, but they are different from typical consumers in several ways. For example, while a typical consumer has more than one victim during its life, parasites as a rule have only one victim per life stage (Lafferty and Kuris, 2002). Also, parasites do not necessarily kill or fully consume their victims. Parasites may also act as prey in a food web, and can be seen as part of trophic interaction in this way as well (Faust, 1975; Marcogliese and Cone, 1997; Johnson et al., 2010; Thieltgets et al., 2013). Inclusion of 47 parasites in an aquatic food web, for example, gave rise to 1093 new interactions of parasites that were prey for other species (Lafferty, 2013). Parasitic interaction can directly or indirectly influence attributes of species in ecological networks, comparable to other non-trophic interactions. The non-trophic interaction that affects attributes of nodes (hosts), and in that way influences the consumer-resource relation, is called "interaction modification" (Kéfi et al., 2012). The attributes could have a direct or indirect influence on the behavior of hosts and non-hosts, handling time of prey, prey preference, assimilation efficiency, conversion efficiency, mortality, reproduction, and growth, and they are common for the different classes of parasites.

Parasites are diverse but the magnitude of this diversity is unknown and it is impossible to estimate the number of species (Dobson et al., 2008). The main characteristic is that they use a host individual's energy for growth, reproduction, and survival. They have, however, very different life histories and sizes. We distinguish microparasites (viruses, bacteria, fungi, protozoa), macroparasites (endoparasites such as helminthes), ectoparasites (fleas and ticks), parasitic castrators, and parasitoids (Kuris and Lafferty, 2000; Lafferty and Kuris, 2002). At one end of the size spectrum, viruses vary in length from 30 to $200 \mathrm{~nm}$. For example, rabies virus has a length of $180 \mathrm{~nm}$ (Baer, 1991). At the other side of the spectrum, tapeworms, such as Diphyllobothrium, vary from $1 \mathrm{~mm}$ to several meters (Faust et al., 1968). Furthermore, the sizes and masses of parasites compared to their hosts are very diverse and depend on the type of parasite. While most microparasites have ratios between $1: 10^{8}$ and $1: 10^{2}$, parasitoids and parasitic castrators are sometimes of mass and size comparable to their host (Lafferty and Kuris, 2002).

Ectoparasites affect their hosts through energy drain by sucking their blood and by activation of a host's immune response with their saliva. This drain of energy can produce subtle subclinical responses, even when these parasites do not by themselves cause disease in their hosts. However, ticks and fleas can also transmit other parasite species, notably microparasites, initiating infection inside of the host that can lead to strong clinical effects, including substantial morbidity, impairing normal ecological functioning, and mortality. In Ngorongoro Conservation Park in 2000 and 2001 there occurred significant mortality among buffaloes, wildebeests, lions, and rhinoceros that had showed infection with Babesia species transmitted by ticks (Nijhof et al., 2003; 
Munson et al., 2008). But parasites carried by ectoparasites can also cause only subtle subclinical effects in host species to which they have strongly adapted.

Subclinical and clinical effects of parasites impact on the overall fitness of the host. Microparasites and macroparasites often negatively influence the fitness of the host, while parasitic castrators and parasitoids reduce fitness of the host to zero (Kuris and Lafferty, 2000; Lafferty and Kuris, 2002). Fitness reduction (e.g., reduced growth and reproduction) of the host originates from the effect of the parasites on the host's ability to feed and on the efficiency of using ingested food for maintenance and production (Anderson and May, 1979; Lafferty et al., 2006). Workers of the bumblebee, Bombus terrestris, challenged with lipopolysaccharides in order to induce their immune system under starvation, reduced survival by 50 to $70 \%$ (Moret and Schmid-Hempel, 2000). Further, multiple parasite infections in North American red squirrels, Tamiasciurus hudsonicus, negatively impact reproductive success due to allocation of the energy toward immune response (Gooderham and Schulte-Hostedde, 2011). Similarly, experimental removal of ectoparasites (mainly fleas) in Columbian ground squirrels, Spermophilus columbianus, led to an increase of female body condition (Neuhaus, 2003). Parasitic castrators and parasitoids extend this effect even more utilizing almost completely the host's energy that is assimilated through trophic interaction directly for its own reproduction and growth, leading to zero reproduction or death for the host itself (Kuris and Lafferty, 2000; Lafferty and Kuris, 2002, 2009; Hechinger et al., 2008).

Parasites can have many different functional roles (Poulin, 1999; Selaković et al., 2014). The difference in the susceptibility of possible hosts, gives to the parasite a role in shaping the population abundance of the host species, thereby affecting the other types of non-trophic interactions. The difference in susceptibility to the malarial parasite, Plasmodium azurophilum, of two species of lizards in the Caribbean plays an important role in their coexistence (Schall, 1992). Some species of parasite affect their host by changing the host's behavioral and physical characteristics and by altering the feeding relationship of the host with its consumers and predators (Moore, 2002). An experiment of three-spined sticklebacks, Gasterosteus aculeatus, that received the same amount of uninfected prey and prey infected with Pomphorhynchus laevis showed significant difference in the predation rate on infected individuals due to the parasite's impact on color and behavior (Bakker et al., 1997). Sometimes parasites lead host species to functional extinction, an example of which will be given below. Many additional interesting examples from the literature that illustrate other functional roles of parasites have been reviewed elsewhere (Selaković et al., 2014).

The above motivates a closer look at how, in ecological theory, parasites of all types can be integrated, and what can then be learned from studying the combined ecological and epidemiological dynamics. There is a large and rich literature on infectious disease dynamics and its mathematical and computational tools and models (see Diekmann et al., 2013 and Heesterbeek et al., 2015, for recent overviews). Mostly, this literature has developed around combinations of one parasite species and one host species. Broadly speaking, there are two modeling approaches depending on the nature of the parasite. If the extent of infection in a host individual and its effects on the host's life history can be quantified at the level of the parasite and is influenced by, or even depends 
on, re-infection, models are in terms of the "degree" of infection (for example, number of hosts carrying $n$ parasites, or the mean parasite load of infected individuals or the environment). These models typically relate to macroparasites. Typically, such parasites and models involve distinct stages in the life cycle, related to different host species or free living in the environment. If the course of infection and its effects on the host are a more or less autonomous process from first successful exposure, models are in terms of generalized and uniform epidemiological states for host individuals (for example, susceptible, latently infected, infectious, immune). Such models typically relate to microparasites.

Work relating to multiple host species interacting with a single parasite species has emerged, but initially ignoring relations within and between host species that were not linked to infection. Only in recent years has there been more substantial effort to regard parasites in systems of multiple host species that also interact ecologically. There is a growing literature, with studies ranging from specific models to more general theory (see Roberts and Heesterbeek, 2012 and the references therein).

In addition to the distinction in approaches in epidemiological models for macro- and microparasites, the added ecological dimension introduces another choice to be made: infectious disease agents can be studied directly or indirectly (Selaković et al., 2014). In the direct approach, parasites are studied as species in food webs, represented by nodes in the web with links to species that are their hosts. In an indirect approach, parasites are studied through their effects on hosts, for example by recognizing different epidemiological states for host individuals of the host species involved, or by recognizing individual hosts with different dynamic infection levels. The indirect approach would combine well with existing epidemiological modeling frameworks for both macro- and microparasites. The direct approach could combine with macroparasitic epidemiological models, especially for systems with a free-living stage of the parasite.

Here, we aim to give an idea of the intricate way in which ecological and epidemiological processes interact in determining dynamic behavior, using an indirect approach. We do so by studying a model for a simple situation for which the ecological dynamics, in the absence of a parasite, are well known. To motivate our model and analysis, we give a few examples of infection in the resource species and its effect on the consumer-resource relationship as an introduction to our next section where we discuss the influence of infection in the resource to the interaction with the consumer. Gerbils, Gerbillus andersoni, affected by the higher abundance of the fleas Synosternus cleopatrae than in nature had higher rates of body mass lost than non-parasitized control individuals (Hawlena et al., 2006). This was probably due to their reduced attention to forage (Raveh et al., 2011). The loss of body mass in gerbils influences their consumers by their need to use more energy to catch additional prey, but on the other side it makes prey more available because of the lack of attention to detect a predator. Further, a nematomorph parasite infects crickets and changes their behavior leading crickets to enter streams and become a new prey connection for predators changing the strength of their neighboring interactions in the trophic network (Sato et al., 2012), where we see the indirect impact of the rest of the trophic network community. Infection in resource can lead to functional extinction of consumer species: the Asian chestnut fungus effectively 
extirpated the American chestnut from eastern US forests, causing the apparent extinction of several phytofagus insects (Anagnostakis, 1987).

We do not claim realism in our model that allows insight into specific disease systems, but at a general level we can discuss the bidirectional influence between a parasite and a consumer-resource relation in different types of ecosystems in terms of energy flow, mirroring Rip and McCann (2011) who looked at non-parasitic systems. We explore the influence of non-trophic parasitic behavior going from aquatic to terrestrial ecosystems. Although terrestrial and aquatic parasites influence their hosts and non-hosts in similar ways, there are distinctions between terrestrial and aquatic environments that influence, for example, parasite biodiversity. Only nine animal phyla are found in terrestrial ecosystems compared to 34 in aquatic ecosystems. This indicates that biodiversity of hosts and parasites may be higher in aquatic ecosystems (McCallum et al., 2004). Further, there are differences in the types of parasites that appear in the two ecosystems. Parasitoids are relatively common for terrestrial ecosystems (Godfray, 1994), while the opposite applies for parasitic castrators (Kuris, 1974). The differences between the two environments and their parasites extends to ways of transmission. Rates of spread of infection in marine ecosystems are higher than those observed in terrestrial ecosystems (McCallum et al., 2003). Also, vertical transmission is very rare in aquatic ecosystems, as well as vector transmission of the diseases (although there are some examples: fireworms spreading Vibrio sp. among corals).

\subsection{Including a Simple Microparasite Affecting Feeding Behavior in a Simple Consumer-Resource Relationship}

Taking into consideration the above examples illustrating how different types of parasites affect their hosts, we analyzed a simple Lotka-Volterra consumer-resource model. Mathematically more sophisticated, and ecologically more realistic, models have been studied, but not in a detailed way exploring the interplay between parameters typically involved in ecology and in epidemiology. Our aim is not to provide maximal realism, but to explore the interplay in the system satisfying the minimum requirements to make it non-trivial.

A simple Lotka-Volterra system, for a consumer interacting with a resource, is used for a broader discussion on stability and energy flux. We use the notation in Rip and McCann (2011), who analyze the stability of the simple system without parasites, and concentrate on "relative energy ratio" (defined below). They regard the largest real part of the eigenvalues of the Jacobian evaluated at the steady state where resource $(R)$ and consumer $(C)$ coexist. The system is given by

$$
\begin{gathered}
\frac{d R}{d t}=r R\left(1-\frac{R}{k}\right)-a C R \\
\frac{d C}{d t}=e a C R-m C
\end{gathered}
$$


(a)

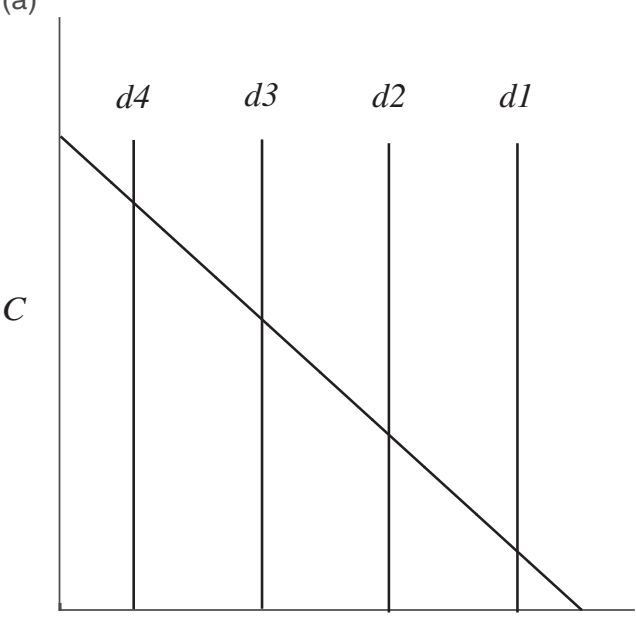

$R$ (b)

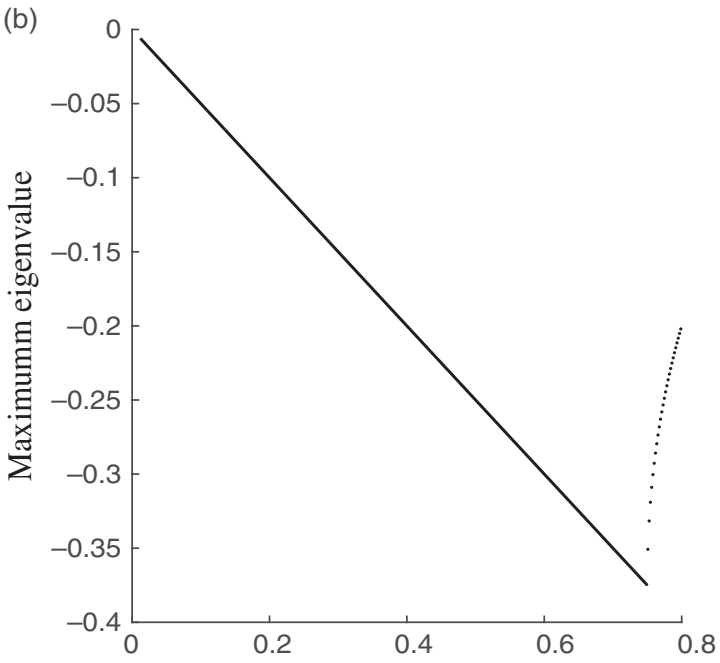

Consumer isocline $(\mathrm{m} / \mathrm{ea})$

Figure 23.1 Simple Lotka-Volterra model analyzed as in Rip and McCann (2011). (a) Increasing $d$, i.e., decreasing the relative energy ratio as defined in Eq. (23.1) in the text, shifts the consumer isocline (vertical line) relative to the resource isocline from right to left $(d l \rightarrow d 4)$. (b) Shifting the consumer isocline to the left, increases the maximum eigenvalue (decreases stability).

with resource growth rate $r$ (biomass time ${ }^{-1}$ ), resource carrying capacity $k$ (biomass), consumption coefficient $a$ (biomass $^{-1}$ time $^{-1}$ ), conversion efficiency $e$ (dimensionless), and consumer mortality $m\left(\right.$ time $\left.^{-1}\right)$. The consumer is assumed not to have alternative sources of food. Rip and McCann (2011) take $r=k=1$ for convenience and we will do the same. The dynamics around the non-trivial steady state $\left(R^{*}, C^{*}\right)$ are governed by the value of a combined parameter that we shall denote by $d:=m /(e a)$, i.e., $d$ (unit: biomass) denotes the $C$-isocline (see Figure 23.1, left graph). We mirror Rip and McCann (2011) and call the "predation rate" $e a$ between the consumer and the resource relative to the consumer loss rate the relative energy ratio $(e a / m=1 / d$ ) (note that Rip and McCann, 2011, use the word "flux," but we prefer to avoid that because it suggests units time ${ }^{-1}$ ). They argue that aquatic ecosystems have a higher relative energy ratio, a high herbivore/ plant ratio and more variable population dynamics, compared to terrestrial ecosystems. Therefore low values of $d$ would relate more to the behavior of an aquatic ecosystem and unstable dynamics, while high values of $d$ would relate more to the behavior of a terrestrial system with a low herbivore/plant ratio and stable population dynamics.

The steady state of the simple model $\left(R^{*}, C^{*}\right)=(d, 1 / a-d / a)$ is stable when it exists, i.e., for $0<d<1$. Because for this simple system the eigenvalues of the Jacobian evaluated at the steady state can be given analytically, one can easily show that the largest real part (and hence the stability) depends on $d$ in a way described in Figure 23.1, right graph. The Jacobian is given by 


$$
\mathbf{J}=\left(\begin{array}{cc}
-d & -a d \\
e(1-d) & 0
\end{array}\right)
$$

If $0<d<4 a e /(1+4 a e)$, the eigenvalues are complex and the largest real part is linearly decreasing in $d$; for $4 a e /(1+4 a e)<d<1$, the eigenvalues are real and a nonlinear increasing function of $d$. So although the non-trivial steady state is stable where it exists, the return time to equilibrium, as provisionally measured by the absolute value of the largest negative real part of the eigenvalues, is a non-linear function of the combined parameter $d$, describing the ecological balance for the consumer of death and recruitment via resource consumption.

This simple system, with clear behavior, is an interesting null model to explore the influence of infectious agents on consumer-resource interaction. We now regard a nonlethal parasite for which only the resource species is a host. We study the dynamics and stability of the parasite-resource-consumer system. In this specific case we model consumer-resource-microparasite interaction. One could model the epidemiology in many different ways but we choose to keep things simple as an initial exploration and allow some analytic tractability. The consumer-resource-microparasite system we study is as follows:

$$
\begin{gathered}
\frac{d R_{s}}{d t}=r R_{s}\left(1-\frac{R_{s}}{k}\right)-a C R_{s}-\beta R_{S} R_{i} \\
\frac{d C}{d t}=e a R_{s} C+q e p a R_{i} C-m C \\
\frac{d R_{i}}{d t}=\beta R_{s} R_{i}-\operatorname{paR}_{i} C
\end{gathered}
$$

Here, $R_{s}$ denotes the susceptible resource population and $R_{i}$ the infected (= infectious) resource population. The transmission rate is denoted by $\beta\left(\right.$ time $\left.^{-1}\right)$. It is the probability per unit of time for one susceptible individual to become infected, i.e., the infection pressure that one infectious individual exerts on susceptible individuals. The dimensionless factors $p>0$ and $q>0$ describe the influence that infection has on the consumption coefficient and conversion efficiency, respectively.

It is important to first note that now we have two different biological points of view for stability. There is ecological stability and epidemiological stability. Ecological stability refers to the balance in the system in terms of intra- and interspecies interaction in the absence of infection; epidemiological stability refers to the balance in the system in terms of the parasite and its hosts. In the absence of the parasite $\left(R_{i}=0\right)$, the system (Eqs. 23.3a-c) is equal to the Lotka-Volterra system $(1)$ in the $\left(R_{s}, C\right)$-plane. The non-trivial steady state $\left(R_{s}^{*}, C^{*}, 0\right)$ of system (3) is ecologically stable in that plane. The first question is when this steady state is also epidemiologically stable, i.e., able to withstand invasion by the infectious agent of the resource. For situations where the agent is able to invade, i.e., where the steady state $\left(R_{s}^{*}, C^{*}, 0\right)$ is epidemiologically unstable, one can then ask the next question under which conditions an endemic steady state is stable, where susceptible and infected resources and consumers all coexist, and 
how does this stability depend on the values of the ecological and epidemiological parameters.

In Roberts and Heesterbeek (2012), the invasion problem is studied for systems where any number of host and non-host species can ecologically interact in a food web, and where a microparasite interacts epidemiologically with its host species. If the uninfected states for all species are listed first (characterizing individuals of non-host species as being always in the uninfected state), followed by the infected states in the same order of species, a general system has a Jacobian matrix of the following form

$$
\mathrm{J}=\left(\begin{array}{ll}
\mathrm{A} & \mathrm{B} \\
\mathrm{D} & \mathrm{T}
\end{array}\right)
$$

where matrix $\mathrm{A}$ is the ecological community matrix, i.e., the Jacobian of the reduced system when the parasite is absent, and where matrix $\mathrm{T}$ is the epidemiological matrix describing transmission among the host species. When $\mathrm{J}$ is evaluated at a given steady state, the eigenvalues of $J$ as usual determine the stability of that steady state. In the case of invasion of an infectious agent (i.e., when looking at the infection-free steady state), we have that matrix $\mathrm{D}$ is the zero matrix, and the eigenvalue problem decouples in the eigenvalues of the community matrix A, now fully governing ecological stability of the infection-free steady state, and the eigenvalues of the epidemiological matrix T, governing the epidemiological stability of the infection-free steady state (Roberts and Heesterbeek, 2012). The characteristic equation of $\mathrm{J}$ is then the product of the characteristic equations of $\mathrm{A}$ and $\mathrm{T}$.

In the system described by Eqs. (23.3a-c), where we assume for convenience that $r=$ 1 and $k=1$, the Jacobian at the infection-free steady state $\left(R_{s}^{*}, C^{*}, 0\right)=(d,(1-d) / a, 0)$ is given by

$$
\mathbf{J}=\left(\begin{array}{ccc}
-d & -a d & -\beta d \\
e(1-d) & 0 & p q e(1-d) \\
0 & 0 & \beta d-p(1-d)
\end{array}\right)
$$

and we see that the ecological stability is governed by matrix (23.2), as expected, and that the epidemiological stability is governed by the one-dimensional matrix $\mathrm{T}=\beta d-p$ $(1-d)$. Hence the infection-free steady state is epidemiologically stable as long as $\beta d-p$ $(1-d)<0$, or when $R_{0}<1$, where

$$
R_{0}:=\frac{\beta d}{p(1-d)}
$$

is the basic reproduction number of the infection system. The basic reproduction number is the average number of new cases of an infection caused by a typical infected individual in a fully susceptible population of hosts in steady state (see Diekmann et al., 2013).

Note that $R_{0}$ in Eq. (23.6) is a combination of ecological and epidemiological parameters because a non-host species (the consumer) influences through ecological 


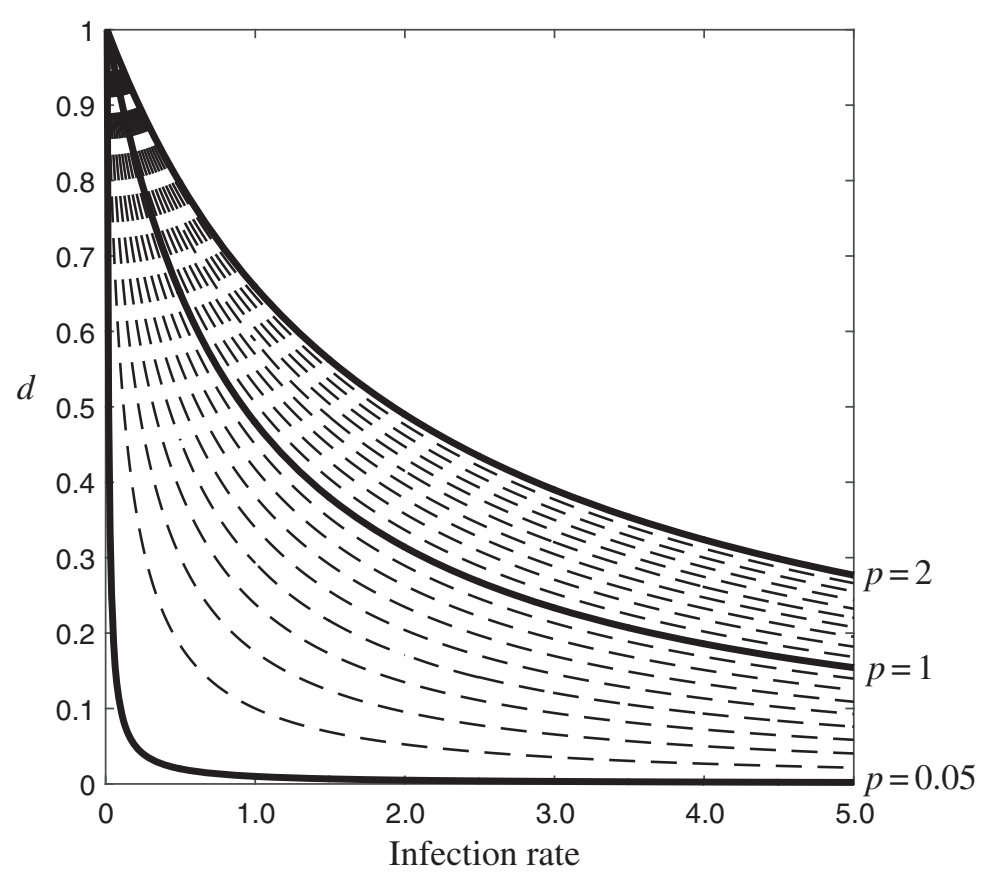

Figure 23.2 The ability of the parasite to invade the consumer-resource system, as a function of infection rate $\beta$, the steady-state population size of the resource in the absence of infection, and for a range of values for the influence of the parasite on the feeding of the host ( $p$; three specific values indicated). For a given value of $p$, the parasite is able to invade the system $\left(R_{0}>1\right)$ for $(\beta, d)$ combinations above the corresponding line.

interaction, epidemiologically relevant aspects of resource individuals (in this case their life expectancy). The biological interpretation is that an infected resource individual is expected to produce $\beta d$ new cases per unit of time during its infectious period with expected length $1 / p(1-d)$. The latter is 1 divided by the probability per unit of time of dying (i.e., by being eaten by a consumer in our model), in the steady state at invasion of the parasite. When $R_{0}>1$, the steady state is epidemiologically unstable and the agent can invade. In Figure 23.2, we show curves in a feasible part of the $(\beta, d)$-plane where $R_{0}=1$, for various values of $p$. We see that, for parasites of limited infectiousness, successful invasion needs more severe ecological effects (smaller values for $p$ ) and higher values for the resource steady state, compared to parasites that induce high infectiousness. Increasing the severity of the ecological effect of the parasite on the consumer-resource interaction (i.e., decreasing the value of $p$ ) increases the area of the $(\beta, d)$-parameter space where the parasite can invade. In ecological terms, if $p$ is small, consumers eat a relatively small proportion of the infected resource population that can hence contact a relatively larger part of the susceptible resource population, leading to more transmission.

Upon successful invasion, the system moves away from the state $\left(R_{s}{ }^{*}, C^{*}, 0\right)$, which is ecologically stable in the $(R, C)$-plane, and moves into the three- 
dimensional space with variables: susceptible resource, consumer, and infectious resource. The system can then converge to the steady state $\left(R_{s}{ }^{*}, C^{*}, R_{i}{ }^{*}\right)$ given by

$$
\begin{gathered}
R_{s}^{*}=\frac{p q-\beta d}{p q+(q-1) \beta} \\
C^{*}=\frac{\beta R_{s}^{*}}{p a} \\
R_{i}^{*}=\frac{d}{p q}-\frac{R_{s}^{*}}{p q}
\end{gathered}
$$

where resource and consumer coexist and the parasite is endemic (endemic steady state).

Some algebra shows that the endemic steady state is feasible, i.e., exists in the sense that all three variables are non-negative, if $\beta$ takes a value in the interval

$$
\frac{p(1-d)}{d}=: \beta_{1}<\beta<\beta_{2}:=\frac{p q}{d}
$$

In this range we will, in the next section, numerically explore the interaction between ecology and epidemiology for the stability of the coexistence of the susceptible resource, the infectious resource, and the consumer population.

\subsection{Numerical Exploration of the Stability of Coexistence}

The analysis of the system described by Eqs. $23.7 \mathrm{a}-\mathrm{c}$ shows how a microparasite that does not produce mortality but only influences the behavior of its host, affects simple consumer-resource systems in different types of environment. In a series of figures, we explore how the stability of the endemic steady state with coexisting consumer, resource, and microparasite changes if we vary epidemiological aspects (the infection transmission rate $\beta$ and the effect of the parasite on the consumption coefficient and the conversion efficiency) and ecological aspects (notably the resource population size $d$ in steady state in the absence of infection, varying between 0.4 and 1 as a result of a mortality variation between 0.3 and 0.75 in steps of 0.05 ). In Table 23.1, we give 10 different regimes of values for the combined parameter $d$ that are explored. By varying $d$ we simulate 10 different "types of environment," and for each of these we vary the

\begin{tabular}{|c|c|c|c|c|c|c|c|c|c|c|}
\hline $\begin{array}{l}\text { Regime } \\
d\end{array}$ & $\begin{array}{l}\text { I } \\
0.400\end{array}$ & $\begin{array}{l}\text { II } \\
0.467\end{array}$ & $\begin{array}{l}\text { III } \\
0.533\end{array}$ & $\begin{array}{l}\text { IV } \\
0.600\end{array}$ & $\begin{array}{l}\text { V } \\
0.667\end{array}$ & $\begin{array}{l}\text { VI } \\
0.733\end{array}$ & $\begin{array}{l}\text { VII } \\
0.800\end{array}$ & $\begin{array}{l}\text { VIII } \\
0.867\end{array}$ & $\begin{array}{l}\text { IX } \\
0.933\end{array}$ & $\begin{array}{l}X \\
1.000\end{array}$ \\
\hline
\end{tabular}
infection transmission rate over a continuous range. Within those combinations for every fixed type of environment $(d)$ and infection transmission rate $(\beta)$, we additionally

Table 23.1 Parameter regimes used for the numerical exploration of model (3) 
vary two other parameters, the conversion efficiency (qe) and the consumption coefficient ( $\mathrm{pa}$ ). We first vary the conversion efficiency by increasing the value of $q$ between 0 and 1 in steps of 0.1 . Finally, for every $(d, \beta, q e)$-combination we vary the consumption coefficient ( $p a$ ) by changing the parameter $p$ between 0 and 2 in steps of 0.1 to simulate the effect of parasite on the feeding behavior of its host, allowing for decreased $(p<1)$ or increased $(p>1)$ consumption of the infected resource by the consumer. We produce graphs of the largest real part of the eigenvalues of the Jacobian matrix, evaluated at endemic steady state, with positive values implying an unstable steady state, and negative values implying stability.

In Figure 23.3, we show stability in the $(\beta, d)$-plane, for a range of values for the epidemiological effects $p$ and $q$. The curves in the left panel of Figure 23.3a indicate where the endemic steady exists and is stable, with various shades of gray indicating the size of the largest real part. Darker shades denote smaller values of the real part of the dominant eigenvalues, and tell us when the system is more resilient to perturbations (a higher return time to equilibrium). White in that figure indicates that either the steady state does not exist in that range of parameter space, or that the steady state is unstable. The results show that the reason an endemic steady state does not exist, for a given combination of parameters $(d, q e, p a)$, depends on the value for the infection rate: at low values for the infection rate the parasite is not able to invade the system under the given conditions, whereas at high values for the infection rate the susceptible resource and consumer interaction cannot sustain the high infection pressure. In Figure 23.3a, right panel, the curves in shade of gray indicate where the steady state exists and is unstable, so here "white" means: the steady state does not exist or is stable). The perturbation of any of the parameters that produce an unstable steady state will easily lead to extinction in either infectious resource or susceptible resource and consumer. Figure 23.3b shows that the endemic steady state exists only in the $\beta$ interval given by Eq. (23.6). In a series of additional figures, we examined the model as follows: (1) without any impact of $q$ and $p$ parameters; (2) with an effect of the disease only on $q$ and only on $p$ separately; and (3) the effect of $p<1$ and $p>1$ on the stability of the system (see Figures S23.1-S23.5 in the appendix).

The stability analyses of the model without any effect of the parasite on conversion efficiency and consumption coefficient of consumer $(p=q=1)$ shows smaller ranges for the infection rate where the species can coexist, compared to the model where these influences are included. That implies the importance of the non-trophic parasitic influences on their hosts and non-hosts for the stability of the system to allow a wider ecological range of interaction conditions suitable for coexistence. The influence of the conversion efficiency parameter and the consumption coefficient parameter separately, showed different effects: when we keep the system without change in consumption coefficient parameter $(p=1)$, lower values of conversion efficiency drive the system sometimes to stable and sometimes to unstable behavior, while when we keep the system without change in conversion efficiency $(q=1)$ lower values of consumption coefficient always lead the system to stability.

If we assume that consumer-resource interaction is affected by the microparasite through both ways of influence ( $p$ and $q$ ), but we are interested in the importance of only 

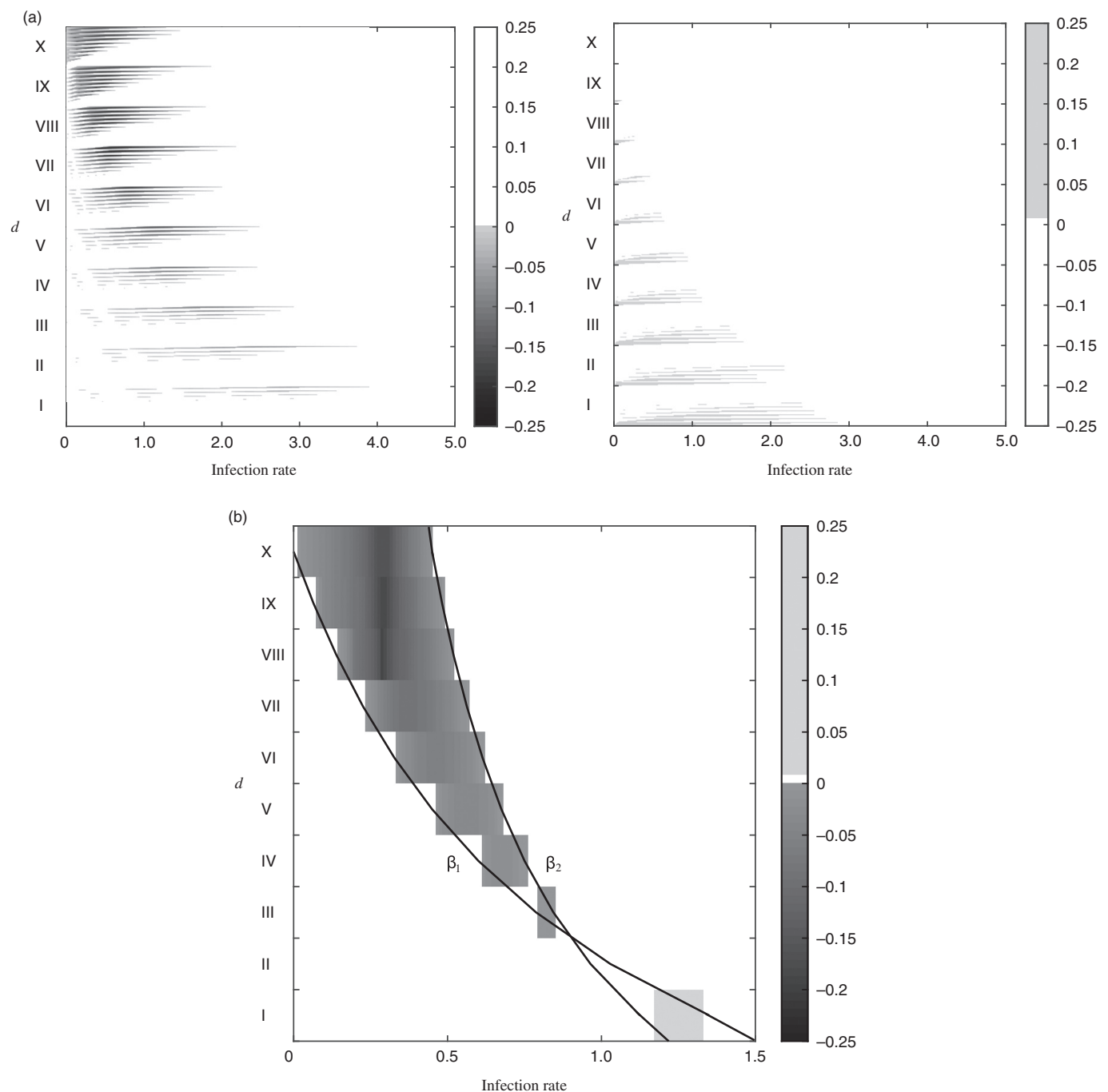

Figure 23.3 Stability analyses of the model $\left(R s^{*}, C^{*}, R i^{*}\right)$. (a) Stability in the $(\beta, d)$-plane, for a range of values for the epidemiological effects $p$ and $q$. The left panel indicates where the endemic steady state exists and is stable, and the right panel indicates where it is unstable. (b) Endemic steady state of the model exists only between $\beta_{1}$ and $\beta_{2}$ given in Eq. (23.6). In this simulation of the toy model we took the values of rates of change for assimilation efficiency $(p=0.9)$ and conversion efficiency $(q=0.5)$.

the effect of the microparasite on the consumption coefficient, we get other interesting insights. The model shows that greater consumption $(p>1)$ of the infected prey gives greater stability to the system and that coexistence of all species can occur with higher infection rates under this condition.

Further, the effects of microparasite on the consumer-resource interaction in different types of environment are examined using the parameter $d$ as in Rip and McCann (2011). 
Table 23.2 Results of percentage of coexistence and stable/unstable distribution ranges of infection rates that support consumer-resource-microparasite coexistence in different types of environment (regimes of predation). Distribution range of infectious rate depends on the consumption coefficient and conversion efficiency parameters.

\begin{tabular}{llll}
\hline$d$ & $\%$ of coexistence & \% of stable & \% of unstable \\
\hline I & $12.9(\beta[0.01-4.34])$ & $23.9(\beta[0.02-4.34])$ & $76.1(\beta[0.01-2.86])$ \\
II & $10.5(\beta[0.01-3.72])$ & $35.5(\beta[0.13-3.72])$ & $64.5(\beta[0.01-2.18])$ \\
III & $9.0(\beta[0.01-3.25])$ & $48.7(\beta[0.01-3.25])$ & $51.3(\beta[0.01-1.67])$ \\
IV & $8.0(\beta[0.01-2.89])$ & $61.9(\beta[0.01-2.89])$ & $38.1(\beta[0.01-1.27])$ \\
V & $7.6(\beta[0.01-2.60])$ & $73.9(\beta[0.01-2.60])$ & $26.1(\beta[0.01-0.95])$ \\
VI & $7.5(\beta[0.01-2.37])$ & $83.7(\beta[0.01-2.37])$ & $16.3(\beta[0.01-0.69])$ \\
VII & $7.6(\beta[0.01-2.17])$ & $91.2(\beta[0.01-2.17])$ & $8.8(\beta[0.01-0.47])$ \\
VIII & $7.9(\beta[0.01-2.00])$ & $95.9(\beta[0.01-2.00])$ & $4.1(\beta[0.01-0.29])$ \\
IX & $8.3(\beta[0.01-1.89])$ & $98.6(\beta[0.01-1.89])$ & $1.4(\beta[0.01-0.13])$ \\
X & $8.7(\beta[0.01-1.73])$ & $100(\beta[0.01-1.73])$ & 0 \\
\hline
\end{tabular}

Following their argumentation, we interpret that going from small to large values of $d$, means that the ecosystem that is modeled changes from "aquatic" to "terrestrial." The stability of the consumer-resource interaction with the infection in the resource if it exists is more often stable in the terrestrial ecosystems, whereas in the aquatic ecosystems it is more frequently unstable. The non-trophic influence of parasites on their hosts and on non-hosts is different in different types of ecosystem. Lowering the conversion efficiency leads to instability in the aquatic ecosystems while in a more terrestrial ecosystem it does not have a destabilizing effect. A higher consumption coefficient ( $p a$ ) in the aquatic ecosystem sustained higher infection rates compared to the more terrestrial ones. The coexistence of consumer-resource-microparasite interaction and the stability distributions of this interaction in different types of ecosystem are presented in Table 23.2 and Figure 23.4. In the table, we show the percentage of the coexistence and infection rates that are supported, as well as the stability distributions with infection rates in different regimes of the steady-state susceptible resource population in the absence of the parasite, $d$ (or $1 /($ relative energy ratio)). Figure 23.4 shows the stability distributions for each of the ten regimes of $d$ (the left panel) and is comparable to Figure 23.1 where stability decreases if we move the consumer isocline $(d)$ to the left. Further, the right panel of Figure 23.4 singles out one regime for $d$ (regime $\mathrm{V}$ ) to better observe the influence of the parasiterelated parameters ( $p$ and $q$ ) on the stability of the interaction.

Table 23.2 and Figure 23.4 suggest that every type of consumer-resource relationship and thus the type of the ecosystem has a specific range of infection rates that can be sustained, and a specific range of parasite-induced characteristics of resource and consumer that allow coexistence in a stable ecosystem of all three species. Additionally, in Table 23.2 we observe that the ecosystems that support the largest ranges for the infection rate and the feeding influences of parasites are those with lower values for $d$, i.e., those with higher relative energy ratios, which we interpret as aquatic ecosystems. 

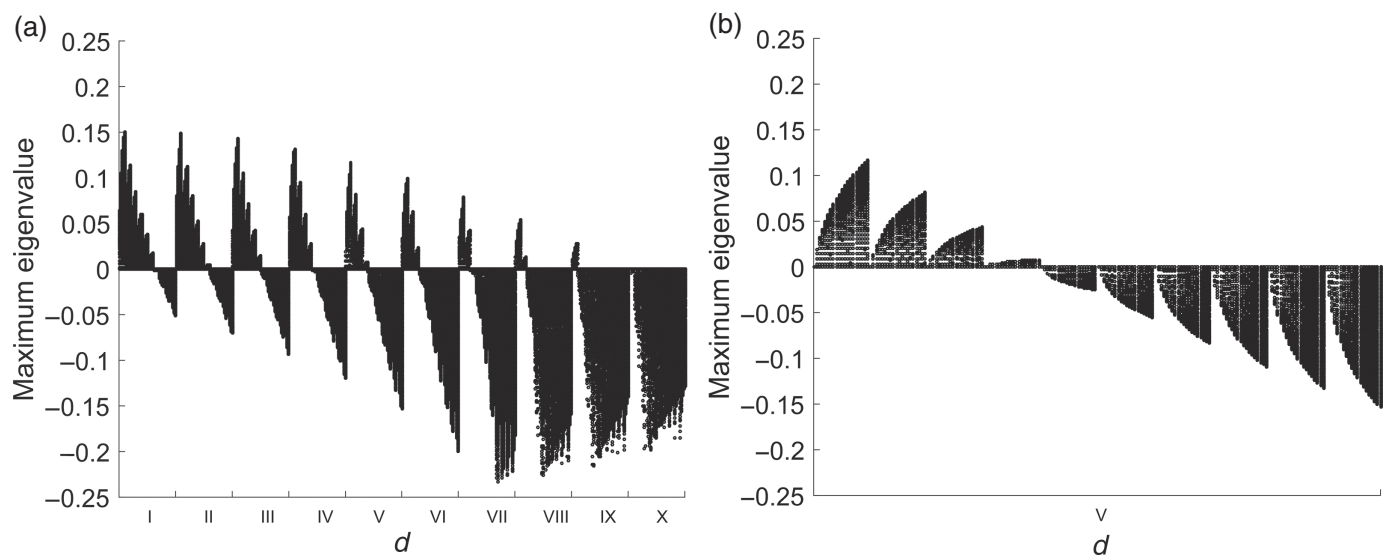

Figure 23.4 Distribution of stable (-)/unstable (+) values in different regimes of $d$. Every dot represents the real part of the dominant eigenvalue for certain combination of parameters $(\beta, d, q e$, $p a$ ). Left panel: all regimes for $d$; right panel: zooming in on regime $\mathrm{V}$ (contains steps of the qe parameter, which incorporate steps of the $p a$ parameter that with a certain infectious rate gives the maximum eigenvalue of the consumer-resource-microparasite system).

\subsection{Discussion}

The research on the inclusion of other than feeding-type interactions in ecological communities, and how these other interactions affect the functioning, structure, and stability of the ecosystems, has developed fast in recent years (Thebault and Fountaine, 2010; Fontaine et al., 2011; Allesina and Tang, 2012; Kéfi et al., 2012; Mougi and Kondoh, 2012, 2014; McQuaid and Britton, 2014; Sauve et al., 2014). Parasitic interaction is one of the first types that was recognized as important in this respect (Lafferty, 1992; Poulin, 1994, 1999; Huxham et al., 1995; Lafferty and Kuris, 2002; Thompson et al., 2004; Lafferty et al., 2006; Kuris et al., 2008; Sukhdeo, 2012; Dunne et al., 2013; Lafferty, 2013; McQuaid and Britton, 2014). One of the proposed ways for studying this particular type of interaction is through the effects parasites have on hosts and on the non-host species their hosts interact with - as non-trophic interaction. This way of incorporating parasites describes their influence via the effect the parasite has on the (physiological/epidemiological) state and behavior of host and non-host species in their ecological network. This is an indirect approach (Kéfi et al., 2012; Selaković et al., 2014), compared to approaches where parasite species are described as biological species, represented directly via their own node in an ecological network where they are linked to nodes representing their host species. Many food-web studies have shown that interaction strengths in food webs are strongly patterned (McCann, 2011), and that both distribution of interaction strengths and the topological structure are important for the stability in ecosystems (Neutel et al., 2002; Allesina and Pascual, 2008). The indirect way of inclusion could be a method to add more accuracy to these analyses by including non-trophic interactions as a real-world simulation. 
Similar consumer-resource models have already discussed the effect of parasites on host behavior and the benefits of consumers foraging on a parasitized resource (Lafferty, 1992), as well as the behavioral effect of trophically transmitted parasites on the dynamics of the consumer-resource relationship (Fenton and Rands, 2006). Here we go one step further with a preliminary interpretation of these effects comparing different types of ecosystem and the range of parasitic influence that are supported by these environments. However, our approach is limited to a model that is very general and we use it only for an initial theoretical discussion, and to highlight phenomena that could occur in relation to stability and coexistence. Moreover, we have concentrated on microparasites that affect only feeding-related behavior of their host without a direct disease-induced effect on the mortality of the host. The advantage of a simple model is that the mutual influence between ecology and epidemiology can be more easily explored. These effects may also occur in more realistic settings, and notably food webs should be explored, rather than the simple two-species food chain in this initial analysis, and for a broader range of parasite types and their influences on hosts.

We included knowledge about the influence of infectious agents on their hosts and non-hosts interactions to the simple consumer-resource interaction using the idea of relative energy ratio (sensu Rip and McCann, 2011, who call it relative energy flux). The idea is that any biological trait that increases the relative energy ratio (predation rate of the consumer relative to its loss term) makes the consumer-resource biomass ratio top heavy and the system less stable. Rip and McCann (2011) examine terrestrial versus aquatic ecosystems and predict that aquatic ecosystems tend to have a higher relative energy ratio and decreased stability relative to terrestrial ecosystems. Our analysis shows how the same idea relates to microparasites in such a setting. One can imagine that every combination of parameters we explored is one type of parasite that influences the consumer-resource relationship in its own specific way. The cross-ecosystem analysis shows that consumer-resource interactions with parasites in different environments are stable within certain ranges of parasitic influence on its host, and therefore for smaller or larger sets of potential parasites. Our analysis suggests that aquatic-like systems, in the above sense, support broader ranges of parasites compared to aquatic ones. This agrees with the observation of a higher biodiversity of parasites and their hosts in aquatic ecosystems (McCallum, 2004). For example, oceans contain an estimated $10^{30}$ virus particles, with $10^{23}$ infections occurring each second (Suttle, 2007). Our analysis also shows that aquatic ecosystems with parasites are more unstable for coexistence of susceptible and infectious resource sub-populations and consumers, compared to terrestrial systems. This conclusion agrees with the discussion on crossecosystem stability from Rip and McCann (2011), for the pure consumer-resource case without parasites.

Indirect inclusion of parasites confirms several general insights. Consumer-resource interaction with infection can be stable over broad ranges of values for epidemiological parameters and of influence on ecological processes. These ranges depend on the ecological characteristics of the consumer-resource system, and parasites can extend the ecological range of coexistence. The analysis gives a clear idea of the importance of 
non-trophic interaction via parasites in ecosystems. Although our model is basic, it does capture the essentials. In the introduction section we discussed many examples of how different parasites affect their hosts, from direct energy drain to indirect change in feeding interaction between resource and consumer. For example, infected resource individuals may be caught less easily by a consumer or more easily. If part of the resource population is infected, consumers spend either more time in search of a suitable prey, or find infected prey using less energy than in the absence of the parasite. Once caught, infected resource individuals may also affect consumers by reduced feeding value. These trait-mediated effects of parasites can be described with conversion efficiency or consumption coefficient parameters in Lotka-Volterra models. Our results show that including non-trophic influences of parasites increases the stability range and coexistence of the consumer-resource-parasite system compared to the system without non-trophic influence of parasite on its host and non-host. For instance, greater consumption of the infected resource increases the stability of the system and supports higher infection rates. With a higher consumption of infected resource consumers control the infection spread in their resource in our basic setting (in line with the healthy herds hypothesis; Packer et al., 2003).

The next step is to expand this theoretical model to a food web that includes many connected consumer-resource relationships, and to explore trait-mediated parasitic impacts on energy flow, strength of interactions, and stability in different types of ecosystem. Parasites included in food webs in an indirect way can increase and decrease the strength of interaction between neighboring species. It would be interesting to see the consequences of such influences even when they are very weak, as such weak links have been shown to play a role in ecosystem stability (Neutel et al., 2002). Because one can hardly observe or measure ecosystems without parasites playing a role (as every living species is a host to probably several types of parasite; Rossiter, 2013), it may be that interaction strengths are importantly moderated by the omnipresence of parasites and that these parasites, even though having very weak effects on individuals, do play a major role in shaping stability and structure in real ecosystems.

\subsection{Summary}

In nature, ecological communities exist as a result of different interactions between species determining structure, functioning, and stability. Empirical as well as theoretical studies are mostly focused on trophic (consumer-resource) interactions and non-trophic interactions separately. Recently, in theoretical and field work, studies started to explore combinations of these interaction types, notably looking at the way infectious disease agents affect consumer-resource relationships in food webs. Here we illustrate such influence by looking at a simple model of a microparasite in a very basic Lotka-Volterra consumer-resource system. We show that even in this simplest of settings one can see a diverse range of subtle changes in system behavior if one lets the main trophic parameters for both host and non-host species be influenced by non-trophic interaction. 


\section{Appendix}

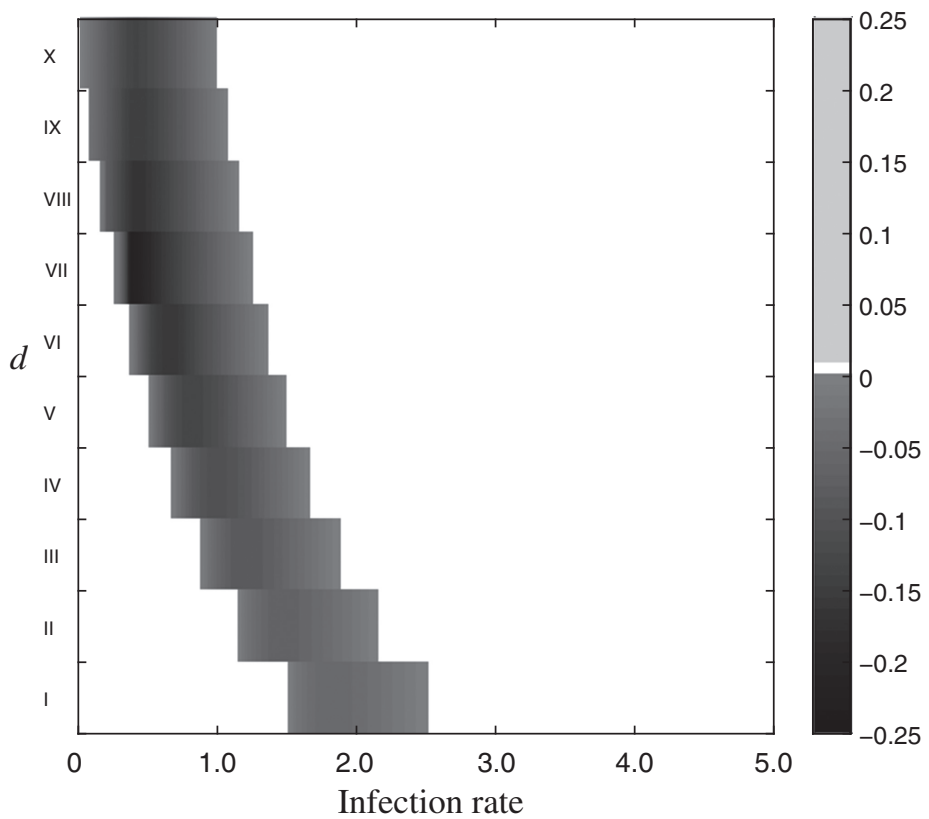

Figure S23.1 The stability analyses of the model without impact of epidemiological parameter $p$ on consumption coefficient $(a)$ of the consumer and epidemiological parameter $q$ on conversion efficiency $(e)$ of the consumer.

(a)

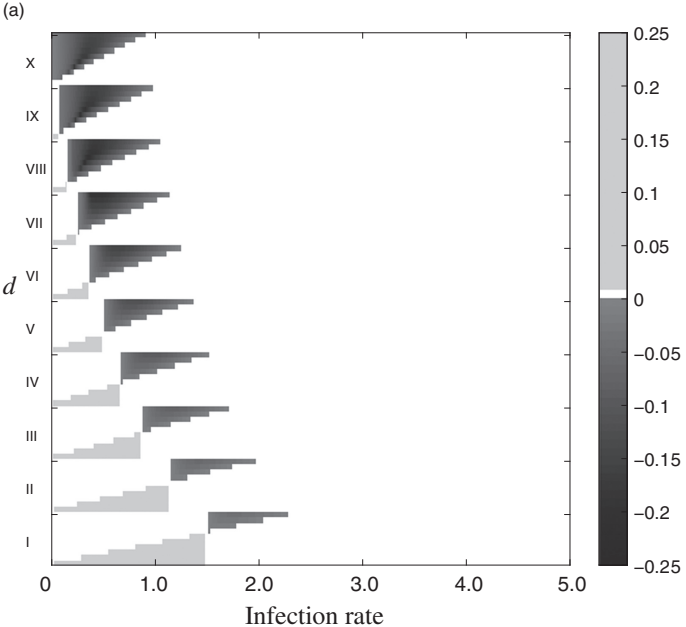

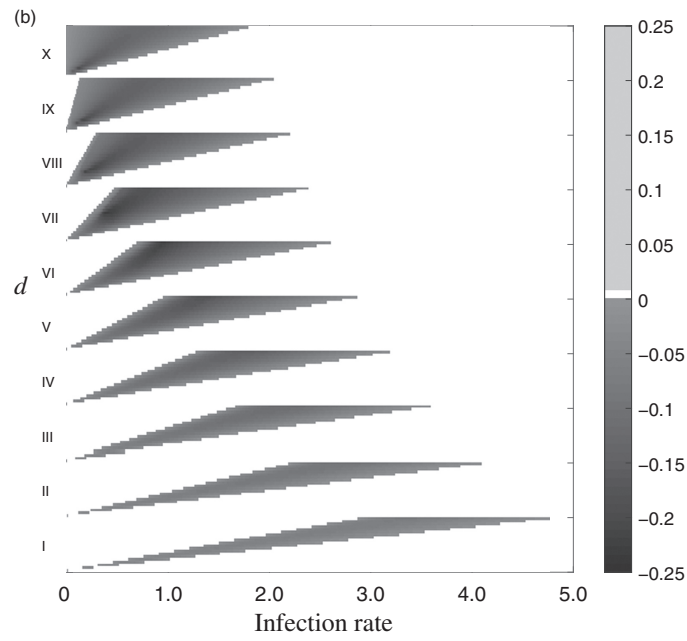

(b)

Infection rate

Figure S23.2 Stability analyses of infection impact on (a) only conversion efficiency $q$ (when the $p$ parameter is fixed) and (b) only consumption coefficient $p$ ( $q$ parameter is fixed). 
(a)

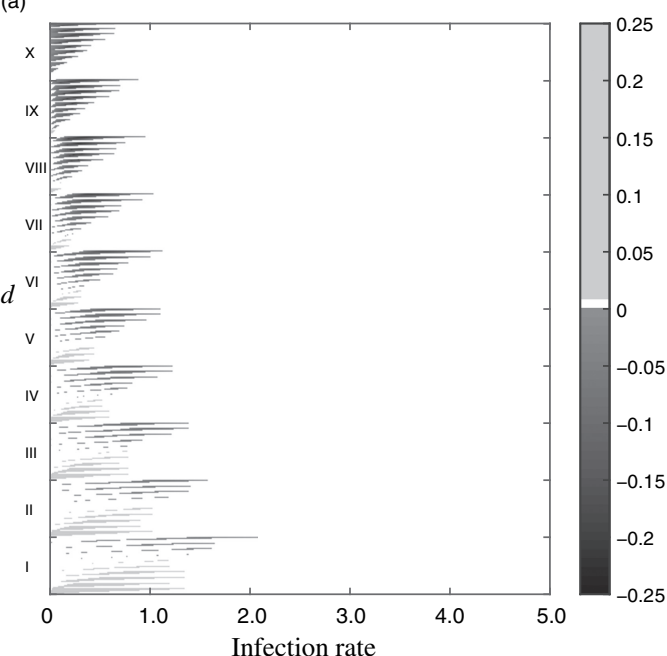

(b)

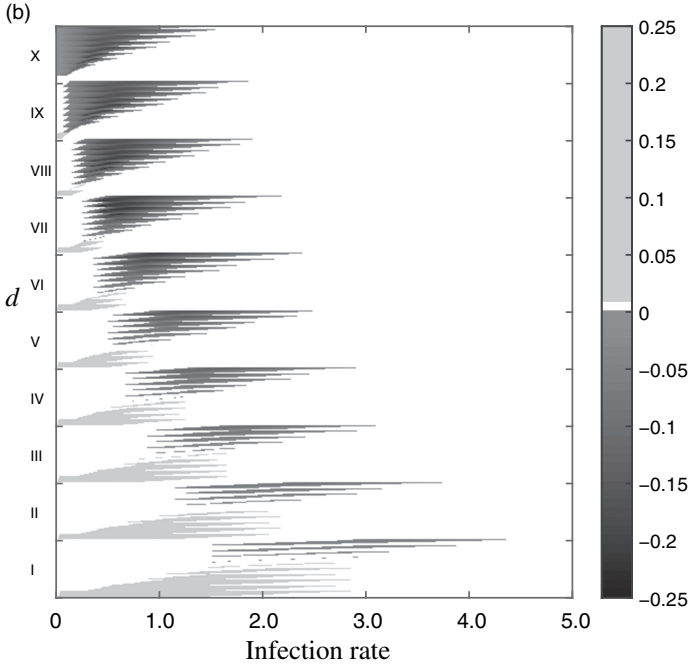

Figure S23.3 Stability analyses for infection impact on consumption coefficient parameter.

(a) Left panel shows the stability analyses with combination of parameters $d$, qe, and $a^{*}(p<1)$, while (b) right panel, shows the stability analyses with combination of parameters $d, q e$, and $a^{*}$ $(p>1)$.

(a)

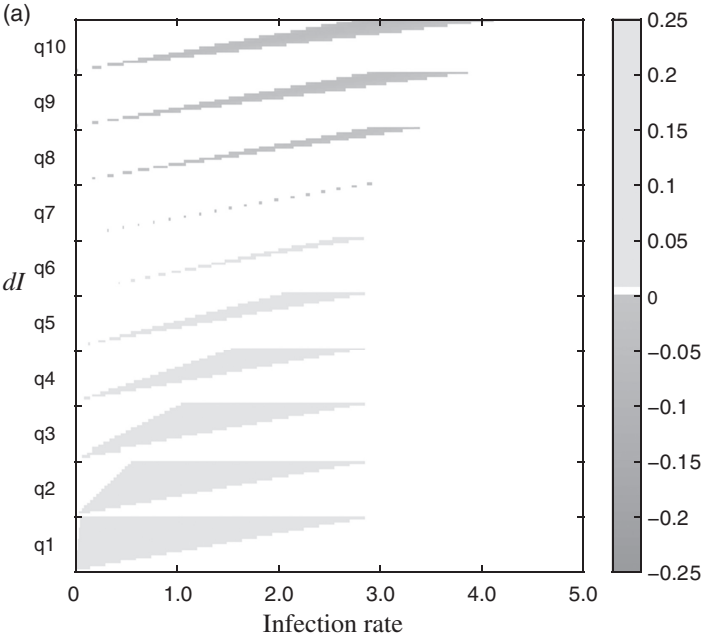

(b)

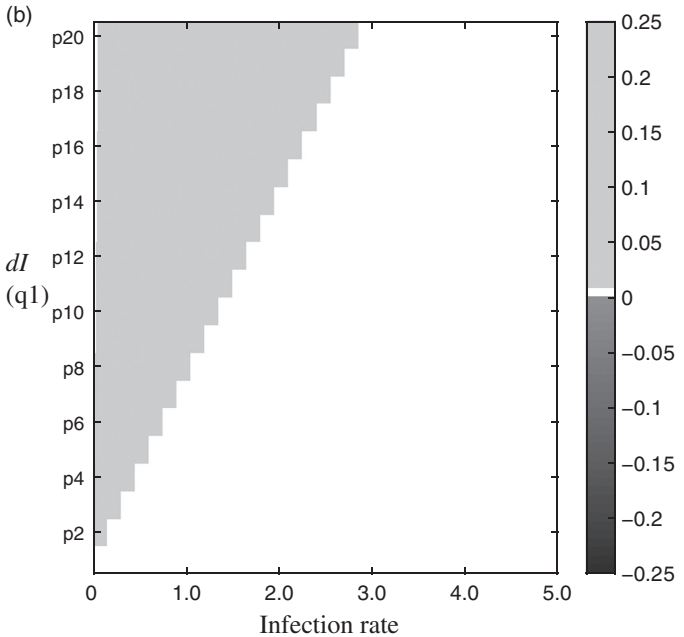

Figure S23.4 Stability analyses of the toy model fixed only on $d I$ where we show the influence of parameters (a) $q$ and (b) $p$ in this situation of energy flux that is compared to energy flux with higher turn-over rates as in aquatic ecosystems. 

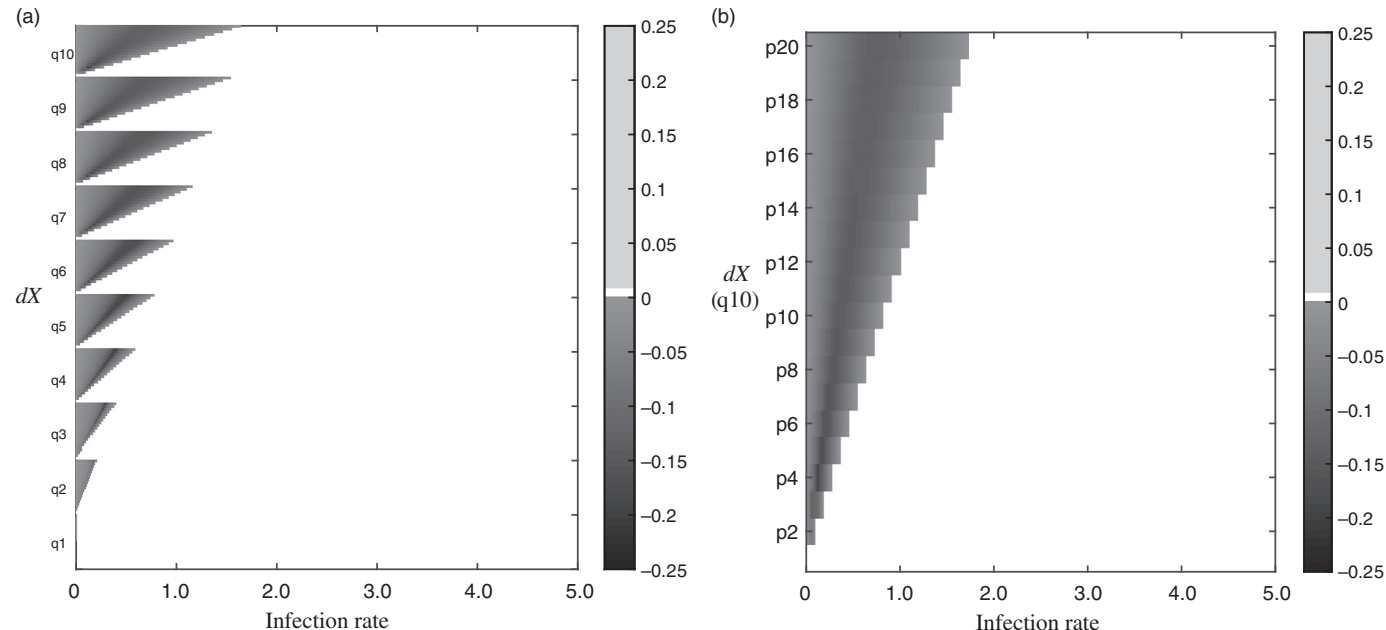

Figure S23.5 Stability analyses of the toy model fixed only on $d X$ where we show the influence of parameters (a) $q$ and (b) $p$ in this situation of energy flux that is compared to energy flux of terrestrial ecosystems.

\section{References}

Allesina, S. and Pascual, M. (2008). Network structure, predator-prey modules, and stability in large food webs. Theoretical Ecology, 1, 55-64.

Allesina, S. and Tang, S. (2012). Stability criteria for complex ecosystems. Nature, 483, 205-208.

Anagnostakis, S. L. (1987). Chestnut blight: the classical problem of an introduced pathogen. Mycologia, 79(1), 23-37.

Anderson, R. M. and May, R. M. (1979). Population biology of infectious diseases: Part I. Nature, 280, 361.

Baer, G. M. (1991). The Natural History of Rabies. Boca Raton, FL: CRC Press.

Bakker, T. C., Mazzi, D., and Zala, S. (1997). Parasite-induced changes in behavior and color make Gammarus pulex more prone to fish predation. Ecology, 78, 1098-1104.

Diekmann, O., Heesterbeek, H., and Britton, T. (2013). Mathematical Tools for Understanding Infectious Disease Dynamics, Princeton, NJ: Princeton University Press.

Dobson, A., Lafferty, K. D., Kuris, A. M., Hechinger, R. F., and Jetz, W. (2008). Homage to Linnaeus: How many parasites? How many hosts? Proceedings of the National Academy of Sciences of the United States of America, 105, 11482-11489.

Dunne, J. A., Lafferty, K. D., Dobson, A. P., et al. (2013). Parasites affect food web structure primarily through increased diversity and complexity. PLoS Biology, 11, e1001579.

Faust, E. C. (1975). The Leishmania parasite of man. In Animal Agent and Vector of Human Disease, ed. E. C. Faust, P. C. Beaver, and R. C. Jung, Philadelphia: Lea \& Febiger, pp. 33-45. 
Fenton, A. and Rands, S. (2006). The impact of parasite manipulation and predator foraging behavior on predator-prey communities. Ecology, 87, 2832-2841.

Fontaine, C., Guimarães, P. R., Kéfi, S., et al. (2011). The ecological and evolutionary implications of merging different types of networks. Ecology Letters, 14, 1170-1181.

Godfray, H. C. J. (1994). Parasitoids: Behavioral and Evolutionary Ecology, Princeton, NJ: Princeton University Press.

Gooderham, K. and Schulte-Hostedde, A. (2011). Macroparasitism influences reproductive success in red squirrels (Tamiasciurus hudsonicus). Behavioral Ecology, 22, $1195-1200$.

Hawlena, H., Khokhlova, I., Abramsky, Z., and Krasnov, B. (2006). Age, intensity of infestation by flea parasites and body mass loss in a rodent host. Parasitology, 133, 187-193.

Hechinger, R. F., Lafferty, K. D., and Kuris, A. M. (2008). Diversity increases biomass production for trematode parasites in snails. Proceedings of the Royal Society B: Biological Sciences, 275, 2707-2714. DOI 10.1098/rspb.2008.0875 [doi].

Heesterbeek, H., Anderson, R. M., Andreasen, V., et al. (2015). Modeling infectious disease dynamics in the complex landscape of global health. Science, 347(6227), aaa4339. DOI 10.1126/science.aaa4339 [doi].

Huxham, M., Raffaelli, D., and Pike, A. (1995). Parasites and food web patterns. Journal of Animal Ecology, 64(2), 168-176. DOI 10.2307/5752.

Johnson, P. T., Dobson, A., Lafferty, K. D., et al. (2010). When parasites become prey: ecological and epidemiological significance of eating parasites. Trends in Ecology and Evolution, 25, 362-371. DOI 10.1016/j.tree.2010.01.005.

Kéfi, S., Berlow, E. L., Wieters, E. A., et al. (2012). More than a meal. . integrating nonfeeding interactions into food webs. Ecology Letters, 15, 291-300.

Kuris, A. M. (1974). Trophic interactions: similarity of parasitic castrators to parasitoids. Quarterly Review of Biology, 49(2), 129-148.

Kuris, A. and Lafferty, K. (2000). Parasite-host modeling meets reality: adaptive peaks and their ecological attributes. In Evolutionary Biology of Host-Parasite Relationships: Theory Meets Reality, ed. R. Poulin and S. Skorping Morand, New York: Elsevier Science, pp. 9-26.

Kuris, A. M., Hechinger, R. F., Shaw, J. C., et al. (2008). Ecosystem energetic implications of parasite and free-living biomass in three estuaries. Nature, 454, 515-518. DOI 10.1038/nature06970.

Lafferty, K. D. (1992) Foraging on prey that are modified by parasites. American Naturalist, 140, 854-867.

Lafferty, K. D. (2013). Parasites in marine food webs. Bulletin of Marine Science, 89, 123-134.

Lafferty, K. D. and Kuris, A. M. (2002). Trophic strategies, animal diversity and body size. Trends in Ecology and Evolution, 17, 507-513.

Lafferty, K. D. and Kuris, A. M. (2009). Parasitic castration: the evolution and ecology of body snatchers. Trends in Parasitology, 25, 564-572.

Lafferty, K. D., Dobson, A. P., and Kuris, A. M. (2006). Parasites dominate food web links. Proceedings of the National Academy of Sciences of the United States of America, 103, 11211-11216. DOI 10.1073/pnas.0604755103.

Levin, S. A., Carpenter, S. R., Godfray, H. C. J., et al. (2009). The Princeton Guide to Ecology. Princeton, NJ: Princeton University Press. 
Marcogliese, D. J. and Cone, D. K. (1997). Food webs: a plea for parasites. Trends in Ecology and Evolution, 12, 320-325.

May, R. M. (1972). Will a large complex system be stable? Nature, 238, 413-414.

May, R. M. (1973). Complexity and Stability in Model Ecosystems. Princeton, NJ: Princeton University Press.

McCallum, H., Harvell, D., and Dobson, A. (2003). Rates of spread of marine pathogens. Ecology Letters, 6, 1062-1067.

McCallum, H. I., Kuris, A., Harvell, C. D., et al. (2004). Does terrestrial epidemiology apply to marine systems? Trends in Ecology and Evolution, 19, 585-591.

McCann, K. S. (2011). Food Webs. Princeton, NJ: Princeton University Press.

McQuaid, C. F. and Britton, N. F. (2014). Parasite species richness and its effect on persistence in food webs. Journal of Theoretical Biology, 364, 377-382.

Moore, J. (2002). Parasites and the Behavior of Animals. Oxford, UK: Oxford University Press.

Moore, J. C. and de Ruiter, P. C. (2012). Energetic Food Webs: An Analysis of Real and Model Ecosystems. Oxford, UK: Oxford University Press.

Moret, Y. and Schmid-Hempel, P. (2000). Survival for immunity: the price of immune system activation for bumblebee workers. Science, 290, 1166-1168. DOI 8972 [pii].

Mougi, A. and Kondoh, M. (2012). Diversity of interaction types and ecological community stability. Science, 337, 349-351. DOI 10.1126/science.1220529 [doi].

Mougi, A. and Kondoh, M. (2014). Adaptation in a hybrid world with multiple interaction types: a new mechanism for species coexistence. Ecological Research, 29, 113-119.

Munson, L., Terio, K. A., Kock, R., et al. (2008). Climate extremes promote fatal co-infections during canine distemper epidemics in African lions. PLoS One, 3, e2545. DOI 10.1371/journal.pone.0002545.

Neuhaus, P. (2003). Parasite removal and its impact on litter size and body condition in Columbian ground squirrels (Spermophilus columbianus). Proceedings of the Royal Society B: Biological Sciences, 270(Suppl 2), S213-S215. DOI 10.1098/ rsbl.2003.0073 [doi].

Neutel, A. M., Heesterbeek, J. A., and de Ruiter, P. C. (2002). Stability in real food webs: weak links in long loops. Science, 296, 1120-1123. DOI 10.1126/science. 1068326.

Nijhof, A., Cludts, S., Fisscher, O., and Laan, A. (2003). Measuring the implementation of codes of conduct: an assessment method based on a process approach of the responsible organisation. Journal of Business Ethics, 45, 65-78.

Odum, E. P. (1971). Fundamentals of Ecology, 3rd edn. Philadelphia, PA: Saunders.

Packer, C., Holt, R. D., Hudson, P. J., Lafferty, K. D., and Dobson, A. P. (2003). Keeping the herds healthy and alert: implications of predator control for infectious disease. Ecology Letters, 6, 797-802.

Pimm, S. (1982). Food Webs. London, UK: Chapman and Hall.

Poulin, R. (1994). Meta-analysis of parasite-induced behavioural changes. Animal Behavior, 48, 137-146.

Poulin, R. (1999). The functional importance of parasites in animal communities: many roles at many levels? International Journal of Parasitology, 29, 903-914.

Raveh, A., Kotler, B. P., Abramsky, Z., and Krasnov, B. R. (2011). Driven to distraction: detecting the hidden costs of flea parasitism through foraging behaviour in gerbils. Ecology Letters, 14, 47-51. 
Rip, J. and McCann, K. (2011). Cross-ecosystem differences in stability and the principle of energy flux. Ecology Letters, 14, 733-740.

Roberts, M. and Heesterbeek, J. A. P. (2012). Characterizing the next-generation matrix and basic reproduction number in ecological epidemiology. Journal of Mathematical Biology, 66(4), 1-20.

Rossiter, W. (2013) Current opinions: zeros in host-parasite food webs: are they real? International Journal for Parasitology: Parasites and Wildlife, 2, 228-234.

Sato, T., Egusa, T., Fukushima, K., et al. (2012). Nematomorph parasites indirectly alter the food web and ecosystem function of streams through behavioural manipulation of their cricket hosts. Ecology Letters, 15, 786-793. DOI 10.1111/j. 1461-0248.2012.01798.x; 10.1111/j.1461-0248.2012.01798.x.

Sauve, A., Fontaine, C., and Thébault, E. (2014). Structure-stability relationships in networks combining mutualistic and antagonistic interactions. Oikos, 123, 378-384.

Schall, J. J. (1992). Parasite-mediated competition in Anolis lizards. Oecologia, 92, $58-64$

Selaković, S., de Ruiter, P. C., and Heesterbeek, H. (2014). Infectious disease agents mediate interaction in food webs. Proceedings of the Royal Society B: Biological Sciences, 281(1777), 20132709. DOI 10.1098/rspb.2013.2709.

Sukhdeo, M. V. (2012). Where are the parasites in food webs? Parasites and Vectors, $\mathbf{5}$, 239. DOI 10.1186/1756-3305-5-239 [doi].

Suttle, C. A. (2007). Marine viruses: major players in the global ecosystem. Nature Reviews Microbiology, 5, 801-812.

Thebault, E. and Fontaine, C. (2010). Stability of ecological communities and the architecture of mutualistic and trophic networks. Science, 329, 853-856. DOI 10.1126/science.1188321 [doi].

Thieltges, D. W., Amundsen, P., Hechinger, R. F., et al. (2013). Parasites as prey in aquatic food webs: implications for predator infection and parasite transmission. Oikos, 122, 1473-1482.

Thompson, R. M., Mouritsen, K. N., and Poulin, R. (2004). Importance of parasites and their life cycle characteristics in determining the structure of a large marine food web. Journal of Animal Ecology, 74, 77-85. 